\title{
THE EFFECTIVENESS OF HIGH INTENSITY INTERVAL TRAINING ON HEART RATE VARIABILITY IN OVERWEIGHT AND OBESITY
}

\author{
Desak Made Wahyu Ariningsih ${ }^{1}$ \\ 1*Program Studi Fisiologi Olahraga, Fakultas Kedokteran, Universitas Udayana \\ Jalan PB Sudirman, Denpasar, Bali, Indonesia \\ Email: desakwahyu@yahoo.com
}

\begin{abstract}
Overweight and obesity are associated with autonomic nervous system dysfunction that is one of the considerations for heart disease, decreased parasympathetic activity, and increased sympathetic activity, which is an unfavorable pattern for the heart. Autonomic nervous system measurement can be done in an invasive manner through the assessment of Heart Rate Variability (HRV). Decreased HRV values in obesity occur due to higher body weight and fat mass. Exercise has been shown to increase vagal modulation, HRV, and reduce sympathetic nerve activity and catecholamine plasma levels. Giving High Intensity Interval Training (HIIT) to overweight and obesity is recommended because it has effectiveness in increasing the work of autonomic nerves through weight loss or body fat mass loss as well as stimulation and inhibition of enzymes that affect the sympathetic and parasympathetic nervous systems performance. The balance of the work system on the sympathetic and parasympathetic nerves will provide a cardio protective effect to reduce the risk of heart disease.
\end{abstract}

Key Words: Exercise; Autonomic Nervous System; Fat Mass; Weight Loss

\section{INTRODUCTION}

The increase in the prevalence of obesity increases the risk of non-communicable diseases, such as cardiovascular disease, musculoskeletal disorders, and several cancers. ${ }^{1}$ The National Clinical Guideline Center recommends that adults should increase their activity levels by doing physical activity for at least 30 minutes with moderate or high intensity, even without weight loss, but at least it can improve health and reduce the risk of cardiovascular disease and type 2 diabetes. ${ }^{2}$ During exercise and recovery, the autonomic nervous system modulates cardiovascular function to meet the metabolic needs of working muscles ${ }^{3}$. The autonomic nervous system consists of sympathetic nerves and nerves parasympathetic whose action is regulated by the medulla oblongata. In this case the sympathetic nerves will increase heart rate, muscle contractility, and conduction velocity through the mediation of $\alpha$ and $\beta$ adrenoreceptors. Meanwhile, the parasympathetic nerves work to lower the heart rate through muscarinic receptors. Control of the autonomic nervous system in the heart is also influenced by baroreceptors, chemoreceptors, tissue metabolism, hormones, habits, and ethnicity ${ }^{4}$ HRV is the variation in the interval between consecutive heartbeats depending on the extrinsic regulation of the heart rate. It is a sensitive and informative method of providing an assessment of changes in autonomic nerve activity in both physiological and pathological conditions ${ }^{5}$.

The imbalance between the sympathetic and parasympathetic nervous systems is one of the considerations in detecting the presence of heart disease, decreased vagal activity (parasympathetic activity), and increased sympathetic activity, which is an unfavorable pattern of autonomic nerve activity. Research by comparing individuals who are obese with individuals who have normal body mass index (BMI) shows a response to increased arterial pressure during exercise, which is an indicator of cardiovascular disease risk ${ }^{6}$. Obesity is associated with lower HRV values and autonomic dysfunction at rest. Decreased HRV values in obesity are associated with higher body weight and fat mass ${ }^{7}$. Decreased vagal tone has also been associated with coronary heart disease and sudden cardiac death. In fact, in experimental clinical studies, it is known that individuals with the greatest decrease in vagal tone and / or increase in sympathetic nerve activity after myocardial infarction, they have a higher risk of experiencing sudden cardiac death ${ }^{3}$.

Losing weight through exercise or restricting calorie intake is highly recommended to increase vagal activity ${ }^{8}$. Exercise has been shown to increase vagal modulation, HRV, and reduce sympathetic nerve activity as well as resting plasma catecholamine levels ${ }^{9}$. Exercise intensity plays an important 
role in regulating substrates utilization, both carbohydrates and fats during exercise. From systematic review studies and meta-analyzes, it is concluded that high-intensity interval training (HIIT) is an alternative exercise with time efficiency that is as effective as moderate-intensity continuous training with increased aerobic capacity in young individuals ${ }^{10}$. Many studies currently show that HIIT can be a strategy to increase functional capacity and reduce the risk of mortality in overweight and obese ${ }^{11}$. Increased exercise intensity can result in a significant increase in vagal activity ${ }^{12}$. Determination of optimal and safe exercise intensity needs to be done in overweight subjects and obesity through assessment of autonomic neural mechanisms. Based on the background described, this study aimed to see the effect of HIIT on increasing modulation of the cardiac autonomic nervous system through HRV analysis in overweight and obese subjects.

\section{METHODS}

In this study, the literature review method was used, namely by conducting studies on several research journals related to HIIT and the cardiac autonomic nervous system through HRV analysis in overweight and obese subjects. The literatures are from electronic database such as PubMed, Pedro, Google Scholar and PEDro. The literatures are search from January $2^{\text {nd }} 2020$ to May $5^{\text {th }} 2020$. The ebook from database also used for the literature. The literatures were searched with key word 'overweight, sedentary, inactive, and obesity', 'high intensity interval training for overweight and obesity' high intensity interval training increases heart rate variability', 'correlation between fat mass with heart rate variability', 'overweight/obesity and autonomic nervous system'. The study that was discussed about overweight/obesity and high intensity interval training and also physical activity that affects autonomic nervous system in overweight or obese subjects used to this study. We performed a hand search of the reference list of retrieved studies to detect manuscript not found by the search in the electronic engines mentioned above. Inclusion criteria: studies were included in this study if they are: 1) in English, 2) reported about the correlation overweight and obesity with heart rate variability, 3) reported about high intensity interval training in overweight and obesity for heart rate variability and autonomic nervous system, 4) study clinical trial, randomized controlled trial, systematic review and meta analysis, review article and experimental study. Exclusion criteria: studies were excluded from this study if they are : 1) didn't report about the result, 2) not suggest about the exercise for heart rate variability and autonomic nervous system in overweight and obese.

\section{DISCUSSION}

a. Association between obesity and heart rate variability (HRV)

Heart Rate Variability (HRV) is a description of the irregularity in heart rate that reflects the effects of the sympathetic and parasympathetic branches and other physiological control mechanisms on heart function ${ }^{13}$. Assessment of the autonomic nervous system regulation using the HRV method can be analyzed through time domain, frequency domain, nonlinear analysis, time-frequency domain, and others. The most frequently used measurement is the time domain, including R-R intervals (SDNN) that represent the variation in the R-R interval at the time of measurement; root mean square of successive differences (RMSDD) that represent the vagal tone and correlating high-frequency (HF) $\mathrm{HRV}$; the percentage of successive normal sinus RR intervals of more than $50 \mathrm{~ms}$ (pNN50) that represents the vagal tone and the measurement of the frequency domain consisting of low frequency (LF) represents the sympathetic nervous activity and high frequency (HF) represents the combination of the sympathetic nervous system and partly parasympathetic; The LF / HF ratio represents the balance of the two autonomic nervous systems, where a decrease in the LF / HF ratio assessment indicates an increase in parasympathetic or a decrease in sympathetic ${ }^{13,14}$.

The resting phase of autonomic nerve dysfunction reflects low vagal cardiac activity characterized by decreased baroreflex sensitivity. The reduced sensitivity of the baroreflex in obesity will interfere with the regulation of the afferent feedback mechanism that plays a role in producing the proper autonomic adjustment of the cardiovascular system when exercise is disrupted by a higher amount of body fat ${ }^{15}$. In addition, excess fat tissue will produce cytokines and pro-inflammatory substances. others that affect autonomic nerve function ${ }^{16}$. Cytokines, such as TNF- $\alpha$ and IL-6, nonesterified free fatty acids, ghrelin, neuropeptide Y, melanocortin and decreased adiponectin and leptin resistance are mediators in the activation of the sympathetic nervous system ${ }^{17}$. Increased nervous 
activity sympathetic induced by leptin can increase lipolysis in white adipose tissue, thermogenesis in brown adipose tissue and increase metabolic activity in liver and skeletal muscle resulting in increased energy expenditure ${ }^{18}$.

Obese subjects had higher baseline HR and blood pressure values compared to eutrophic types. In autonomic nerve modulation, significant reductions were observed in the RMSSD, SD1 index, and HF values. The obese group showed decreased vagal activity and decreased SDNN, SD2 levels and decreased overall variability. The high LF (nu) index value and the decrease in the mean RR in obese group in this case indicate the dominance of sympathetic nerves and decreased activity of the vagal or parasympathetic nerves. ${ }^{19}$ This decrease in variability is a cause for concern because it is associated with an increased risk of heart disease due to decreased adaptation of the heart to stressful situations ${ }^{3}$.

b. Effect of high intensity interval training on heart rate variability

HIIT is high intensity exercise and is done repeatedly. It consists of alternating periods of high intensity exercise involving 30-300 seconds of aerobic exercise at $85-100 \%$ of maximum rate oxygen consumption $\left(\mathrm{VO}_{2} \max \right)$ followed by periods of rest or low intensity exercise of the same or shorter duration to allow subjects to allocate greater time to high intensity rather than continuous exercises ${ }^{20}$. A systematic review conducted by Thivel at al (2018) concluded that HIIT can significantly reduce blood pressure, insulin resistance, triglycerides, and elevated HDL-C ${ }^{21}$. HIIT is as good as Moderate Intensity Continuous Training (MICT) in reducing fat mass and abdominal circumference in obese subjects. Furthermore HIIT requires a shorter duration of time for these outcomes if compared to MICT 22.

A systematic review research conducted by Costa et al in 2019 aims to determine the modulation of the sympathetic and parasympathetic nervous systems after weight loss through exercise and diet. One experimental study and 14 observational studies presented the LF value which is a description of the sympathetic nervous system, where the result of some parasympathetic nerves is a decrease in the LF value. HF was measured in one experimental study and found to be significantly improved immediately after the first week of exercise intervention. The study concluded that giving exercise increased the HRV value after weight loss in subjects compared to diet alone. In this case, variations in changes in the autonomic nervous system in obesity are caused by changes in body weight after periodic exercise. Weight gain is generally associated with sympathetic nerve dominance, but it is currently unclear whether the mechanism of dominance is acquired directly or through parasympathetic inhibition ${ }^{23}$.

A randomized control trial conducted by Heydari in 2013 on young men with a mean BMI of $28.7 \pm 3.1 \mathrm{~kg} \mathrm{~m}-2$ who were given HIIT intervention (cycle ergometer) 3 times a week for 12 weeks. HRV was analyzed in supine for 30 minutes with Polar Heart Rate Monitor to get the RR interval then analyze by Polar ProTrainer 5 software. The result showed an increase in at the HF, RMSDD, and pNN50 values where these results reflect an increase in parasympathetic nerve cardiac activity ${ }^{33}$. This research also conclude that HIIT follow by brief moderate intensity enhance cardiac parasympathetic nerve outflow. Increasing RMSDD index is correlated with VO2max enhancement ${ }^{24}$.

A randomized controlled trial study by Phoemsapthawee et al in 2019 to determine the response of HRV and cardiorespiratory fitness in the intervention group by providing a combination of continuous moderate intensity aerobic exercise (fast walking, jogging, ball games, or swimming) and high intensity interval training with resistance training with a frequency of 2 times a week for 12 weeks. While the control group did not receive exercise, only recommended diet and physical activity. The results showed a decrease in body anthropometry and an increase in VO2max. In the measurement of $\mathrm{HRV}$, there was a significant increase in the value of HF, while resting HR, LF, LF / HF ratio, and SD2 after 12 weeks of treatment in the intervention group decreased. This study concluded that HRV was increased due to decreased fat mass and increased aerobic fitness (VO2max) in obese subjects. Subjects with a higher sympathovagal balance had greater increases in physical fitness (VO2max). Increased parasympathetic and decreased sympathetic outflow to the heart elicited by exercise training is typically considered a factor in cardio protection. These findings appeared to support a concept of cardiovascular protection that regular physical activity could reduce mortality and morbidity of $\mathrm{CVD}^{25}$.

A randomized controlled trial study by Rodrigues et al in 2020 was conducted on 70 overweight and obese subjects who were given HIT treatment with different intensities and volumes. The treatment 
that are given in three groups is 1-high-intensity interval training (HIIT) $(n=25,1 \times 4$ min bout at 85 95\%HR peak, $3 \times /$ week), 4-HIIT ( $n=26,4 \times 4$ min bouts at $85-95 \%$ HR peak, interspersed with 3 min of recovery at 50-70\%HR peak, $3 \times /$ week), and moderate continuous training (MCT) $(n=19,30 \mathrm{~min}$ at 60-70\%HR peak, 5x/week). The result of the this study find that 4-HIIT and MCT showed positive outcomes for almost all variables (LF, RMSSD, SD2, and, SD1 index) while 1-HIIT had a positive influence only on SD2 index. An increase in the SD1index means an increase in parasympathetic activity, while an increase in the SD2 index means a decrease in sympathetic activity. This research concluded that the adaptation of the cardiac autonomic nervous system through HRV analysis was related to receptor variants, $\beta 2$ adrenergic (ADRB2) after 16 weeks of HIIT. The results obtained from this study show that genetic characteristics have an influence on the body's adaptation in long term aerobic exercise. The increase in parasympathetic activity is obtained by increasing the LF value ${ }^{26}$.

A randomized controlled trial study by Alansare et al in 2018 compared the effects of two different exercise protocols (HIIT vs. MICT) on heart rate variability in physically inactive adults concluded that 8 HIIT sessions were able to improve sympathetic and parasympathetic nerve balance where the data of this study were analyzed using the LF / HF ratio assessment. The increase in the balance of the sympathetic and parasympathetic nerves occurs because the high intensity of exercise will activate the parasympathetic nerves. It is concluded that, exercise intensity $\geq 80 \%$ heart rate peak (HRpeak) will increase the value of the frequency domain. However, exercise intensity $\leq 80 \%$ HRpeak will not affect the frequency domain value. In this study, the SDNN value (number of R-R intervals) was increased, indicating a decrease in resting heart rate. This indicates an early adaptation to regular exercise. This adaptation is brought about by the sinoatrial node (SA node) adaptation. This research contradicts with past research that was done by Heydari (2013), the resting RMSSD value does not show an increase like the SDNN value, which indicates that a decrease in resting heart rate can be achieved through a decrease in SA node activity without improvement in the autonomic nervous system $^{27}$.

Research that has been conducted has shown that HIIT can reduce levels of catecholamines, betaadrenergic receptors, and levels of angiotensin $\mathrm{II}^{28}$. Research that has been conducted has shown that HIIT can reduce levels of catecholamines, betaadrenergic receptors, and levels of angiotensin II ${ }^{29}$. Research by Holloway in 2015 showed that angiotensin II levels decreased significantly in animal models undergoing HIIT treatment. In addition, other studies have shown that after being given HIIT, there is a decrease in renin-angiotensin levels which is characterized by reduced angiotensin-converting enzymes, angiotensin receptors, and renin receptors ${ }^{30,31}$. Furthermore, administering HIIT interventions also leads to increased levels of nitric oxide (NO) and is a potential mediator that can increase cardiac autonomic modulation stimulated after exercise ${ }^{32}$. NO in this case has an indirect effect on inhibiting sympathetic influence and plays a role in increasing parasympathetic activity ${ }^{33}$. Studies conducted on animal and human subjects have revealed that increased NO levels are associated with increased vagal or parasympathetic activity ${ }^{33}, 34$. Wisloff et al have proven that HIIT can cause fluctuations between high and low intensity, thereby triggering a greater response at the cellular and molecular levels. In addition, HIIT also has benefits in reducing the number of reactive oxygen species and increasing the activity of superoxide dismutase and glutathione peroxidase ${ }^{35}$. A study with patient subjects who had coronary artery bypass graft (CABG) showed that HIIT can increase HRV by increasing HF, SDRR, and RMSSD, as well as reducing the LF and LF / HF ratio. However, the mechanism underlying this effect is not clear ${ }^{36}$.

But on the other hand, research conducted by James et al (2012) on healthy subjects stated that high-intensity training with the interval training type had an effect on decreasing the value of the parasympathetic nerve indicators (SDNN, RMSSD, pNN50, HF, HF (nu)). In addition, this type of exercise also causes an increase in the value for the sympathetic nerve indicator (LF (nu)) 1 hour after the training session. This change is followed by a decrease in systolic blood pressure. This increase in modulation of the sympathetic nerves has an effect on the increased risk of heart disease in highintensity exercise. Conditions will begin to return to normal as in the pre-workout phase after less than 24 hours and will persist 48 hours to 72 hours after exercise ${ }^{37}$. Therefore, training with lower intensity at the start of exercise is recommended to be done first to provide physiological adaptations to the body as preparation before training with a higher intensity. 


\section{CONCLUSION}

High Intensity Interval Training (HIIT) is one of the exercises that can be given to overweight and obesity subjects. This exercise aims to increase the modulation of the autonomic nervous system in the heart. An increase in HRV values will be obtained after continuous HIIT through the mechanism of decreasing body weight or body fat mass, as well as due to stimulation and inhibition of enzymes that affect the performance of the sympathetic and parasympathetic nervous systems. The balance of the work system on the sympathetic and parasympathetic nerves will then provide a cardio protective effect which functions to reduce the risk of heart disease. Adaptation to high intensity exercise for obesity conditions requires further research so that the provision of optimal exercise effectiveness can be appropriately carried out.

\section{REFERENCES}

1. World Health Organization (WHO). 2020. Childhood Overweight and Obesity. (https://www.who.int/dietphysicalactivity/childhood/en/). Diakses 29 Mei 2020

2. National Health Service (NHS). 2019. Obesity Causes. (https://www.nhs.uk/conditions/obesity/causes/). Diakses 1 Juli 2020

3. Nishime EO, Cole CR, Blackstone EH, Pashkow FJ, Lauer MS. Heart Rate Recovery And Treadmill Exercise Score As Predictors Of Mortality In Patients Referred For Exercise ECG. JAMA. 2000;284:1392-98.

4. Triposkiadis F, Karayannis G, Giamouzis G, Skoularigis J, Louridas G and Butler J: The sympathetic nervous system in heart failure physiology, pathophysiology, and clinical implications. J Am Coll Cardiol. 2009. 54:1747-62.

5. Taralov ZZ, Kiril VT, Stefan SK. Heart Rate Variability as a Method for Assessment of the Autonomic Nervous System and the Adaptations to Different Physiological and Pathological Conditions. Folia Medica. 2016;57(3-4): 173-180.

6. Dipla K, Zafeiridis A, Koidou I, Geladas N, Vrabas IS. Altered hemodynamic regulation and reflex control during exercise and recovery in obese boys. American Journal of Physiology. 2010;299(6):209096.

7. Koenig J, Jarczok MN, Warth M, Ellis RJ, Bach C, Hillecke TK. Body Mass Index Is Related To Autonomic Nervous System Activity As Measured By Heart Rate Variability, A Replication Using Short Term Measurements. The Journal of Nutrition Health and Aging. 2014;18:300-2.

8. Tian Y, Huang C, He Z, Hong P, Zhao J. Autonomic function responses to training: correlation with body composition changes. Physiol Behav. 2015;151:308-313.

9. Gademan MG, Swenne CA, Verwey HF, van der Laarse A, Maan AC, van de Vooren H. Effect Of Exercise Training On Autonomic Derangement And Neurohumoral Activation In Chronic Heart Failure. J Card Fail. 2007;13:294- 303.

10. Gist NH, Fedewa MV, Dishman RK, Cureton KJ. Sprint Interval Training Effects On Aerobic Capacity: A Systematic Review And Meta-Analysis. Sports Med. 2014;44: 269-79.

11. Tjønna AE, Leinan IM, Bartnes AT, Jenssen BM, Gibala MJ. Low- And High-Volume Of Intensive Endurance Training Significantly Improves Maximal Oxygen Uptake After 10-Weeks Of Training In Healthy Men. PLoS One. 2013;8: e65382.

12. El Agaty SM, Kirmani A, Labban, E. Heart Rate Variability Analysis During Immediate Recovery From Exercise In Overweight/Obese Healthy Young Adult Females. Ann Noninvasive Electrocardiol.2016;22:e12427

13. Novani N, Prihatmanto A. Heart Rate Variability Frekuensi Domain Untuk Deteksi Stres Mental Dan Influenza Menggunakan SVM Classifier. [Thesis]. Bandung: Institut Teknologi Bandung: 2016.

14. Laborde S, Mosley E, Thayer JF. Heart Rate Variability and Cardiac Vagal Tone in Psychophysiological Research-Recommendations for Experiment Planning, Data Analysis, and Data Reporting. Front. Psychol. 2017;8:213

15. Esco MR, Williford HN. Race influences the relationship be- tween aerobic power and heart rate recovery. Journal of Sports Medicine and Physical Fitness. 2013;53:583-587.

16. Kaufman CL, Kaiser DR, Kelly AS, Dengel JL, Steinberger J, Dengel DR. Diet revision in overweight children: effect on autonomic and vascular function. Clinical Autonomic Research. 2008;18(2):105-8.

17. Prado DM, Silva AG, Trombetta IC, Ribeiro MM, Guazzelli IC, Matos LN, et al. Exercise training associated with diet improves heart rate recovery and cardiac autonomic nervous system activity in obese children. International journal of sports medicine. 2010;31(12):860-5. 
18. Lambert EA, Straznicky NE, Dixon JB, Lambert GW. Should the sympathetic nervous system be a target to improve cardiometabolic risk in obesity? American journal of physiology Heart and circulatory physiology. 2015; 309(2):244-58.

19. Rossi RC, Vanderlei LCM, Gonsalves ACCMR, Yamada KMH, da Silva NT, de Abreu LC. Impact of Obesity on Autonomic Modulation, Heart Rate and Blood Pressure in Obese Young People. Autonomic Neuroscience. 2015;193: 138-41

20. Guiraud T, Labrunee M, Gaucher-Cazalis K, Despas F, Meyer P, Bosquet L, et al. High-intensity interval exercise improves vagal tone and decreases arrhythmias in chronic heart failure. Med Sci Sports Exerc. 2013; 45(10):1861-7.

21. Wewege M, van den Berg R, Ward RE, Keech A. The effects of high-intensity interval training vs. moderate-intensity continuous training on body composition in overweight and obese adults: a systematic review and meta-analysis. Obes Rev. 2017;18(6):635-646.

22. Thivel D, Masurier J, Baquet G. High-intensity interval training in overweight and obese children and adolescents: systematic review and meta-analysis. J Sports Med Phys Fitness. 2019;59(2):310-324

23. Costa J, Moreira A, Moreira P, Delgado L, Silva D. Effects of weight changes in the autonomic nervous system: A systematic review and meta-analysis. Clinical Nutrition. 2020; 38(1):110-126.

24. Heydari M, Boutcher YN, Boutcher SH. High-intensity intermittent exercise and cardiovascular and autonomic function. Clin Auton Res. 2013;23(1):57-65.

25. Phoemsapthawee J, Piyapong P, Naruemon L. Heart rate variability responses to a combined exercise training program: correlation with adiposity and cardiorespiratory fitness changes in obese young men. Journal of exercise rehabilitation. 2019:15(1): 114.

26. Rodrigues JAL, Ferrari GD, Trapé ÁA. $\beta 2$ adrenergic interaction and cardiac autonomic function: effects of aerobic training in overweight/obese individuals. Eur J Appl Physiol.2020;120, 613-24.

27. Alansare A, Alford K, Lee S, Church T, Jung HC. The Effects of High-Intensity Interval Training vs. Moderate-Intensity Continuous Training on Heart Rate Variability in Physically Inactive Adults. Int $J$ Environ Res Public Health. 2018;15(7):1508.

28. Ciolac EG, Bocchi EA, Bortolotto LA, Carvalho VO, Greve JM, Guimaraes GV. Effects of high- intensity aerobic interval training vs. moderate exercise on hemodynamic, metabolic and neuro- humoral abnormalities of young normotensive women at high familial risk for hypertension. Hypertens Res 2010; 33(8): 836-43.

29. Fernandes T, Hashimoto NY, Magalhaes FC, Fernandes FB, Casarini DE, Carmona AK, et al. Aerobic exercise training-induced left ventricular hypertrophy involves regulatory MicroRNAs, decreased angiotensin-converting enzyme- angiotensin ii, and synergistic regulation of angiotensin-converting enzyme 2-angiotensin. Hypertension. 2011; 58(2): 182-9.

30. Holloway TM, Bloemberg D, da Silva ML, Simpson JA, Quadrilatero J, Spriet LL. High intensity interval and endurance training have opposing effects on markers of heart failure and cardiac remodeling in hypertensive rats. PLoS One. 2015; 10(3): e0121138.

31. de Oliveira SG, Dos Santos Neves V, de Oliveira Fraga SR, Souza-Mello V, Barbosa-da-Silva S. Highintensity interval training has beneficial effects on cardiac remodeling through local renin- angiotensin system modulation in mice fed high-fat or high-fructose diets. Life Sci. 2017;189: 8-17.

32. Izadi MR, Ghardashi Afousi A, Asvadi Fard M, Babaee Bigi MA. High-intensity interval training lowers blood pressure and improves apelin and NOx plasma levels in older treated hypertensive individuals. J Physiol Biochem. 2018;74(1): 47-55.

33. Munk PS, Staal EM, Butt N, Isaksen K, Larsen AI. High-intensity interval training may reduce in-stent restenosis following percutaneous coronary intervention with stent implantation A randomized controlled trial evaluating the relationship to endothelial function and inflammation. Am Heart J. 2009; 158(5): 734-41.

34. Massion PB, Dessy C, Desjardins F, Pelat M, Havaux X, Belge C, et al. Cardiomyocyte-restricted overexpression of endothelial nitric oxide synthase (NOS3) attenuates beta-adrenergic stimulation and reinforces vagal inhibition of cardiac contraction. Circulation. 2004; 110(17): 2666-72.

35. Wisloff U, Stoylen A, Loennechen JP, Bruvold M, Rognmo O, Haram PM, et al. Superior cardiovascular effect of aerobic interval training versus moderate continuous training in heart failure patients: A randomized study. Circulation. 2007; 115(24): 3086-94.

36. Ghardashi-Afousi A, Holisaz MT, Shirvani H, Pishgoo B. The effects of low-volume high-intensity interval versus moderate intensity continuous training on heart rate variability, and hemodynamic and echocardiography indices in men after coronary artery bypass grafting: A randomized clinical trial study. ARYA Atheroscler. 2018; 14(6): 260-71.

37. James D, Munson S, Maldonado-Martin S, De Ste Croix M. Heart Rate Variability: Effect of Exercise Intensity on Post-exercise Response. Research Quarterly for Exercise and Sport. 2012;83(4): 533-39. 
